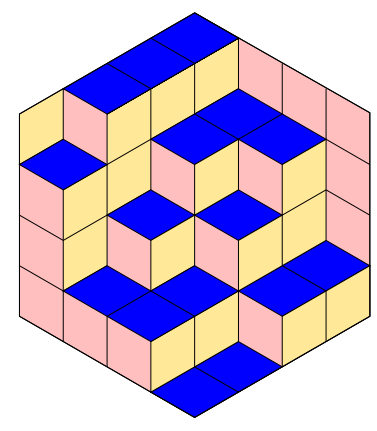

ALGEBRAIC COMBINATORICS

Darcy Best \& Ian M. Wanless

Parity of transversals of Latin squares

Volume 3, issue 2 (2020), p. 539-557.

<http://alco.centre-mersenne.org/item/ALCO_2020__3_2_539_0>

(c) The journal and the authors, 2020.

Some rights reserved.

(c) BY This article is licensed under the

Creative Commons ATtribution 4.0 InTERnational License.

http://creativecommons.org/licenses/by/4.0/

Access to articles published by the journal Algebraic Combinatorics on the website http://alco.centre-mersenne.org/ implies agreement with the Terms of Use (http://alco.centre-mersenne.org/legal/).

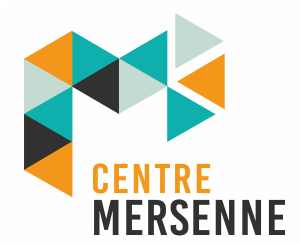

Algebraic Combinatorics is member of the Centre Mersenne for Open Scientific Publishing www.centre-mersenne.org 


\title{
Parity of transversals of Latin squares
}

\author{
Darcy Best \& Ian M. Wanless
}

\begin{abstract}
We introduce a notion of parity for transversals, and use it to show that in Latin squares of order $2 \bmod 4$, the number of transversals is a multiple of 4 . We also demonstrate a number of relationships (mostly congruences modulo 4) involving $E_{1}, \ldots, E_{n}$, where $E_{i}$ is the number of diagonals of a given Latin square that contain exactly $i$ different symbols.

Let $A(i \mid j)$ denote the matrix obtained by deleting row $i$ and column $j$ from a parent matrix $A$. Define $t_{i j}$ to be the number of transversals in $L(i \mid j)$, for some fixed Latin square $L$. We show that $t_{a b} \equiv t_{c d}$ mod 2 for all $a, b, c, d$ and $L$. Also, if $L$ has odd order then the number of transversals of $L$ equals $t_{a b} \bmod 2$. We conjecture that $t_{a c}+t_{b c}+t_{a d}+t_{b d} \equiv 0 \bmod 4$ for all $a, b, c, d$.

In the course of our investigations we prove several results that could be of interest in other contexts. For example, we show that the number of perfect matchings in a $k$-regular bipartite graph on $2 n$ vertices is divisible by 4 when $n$ is odd and $k \equiv 0 \bmod 4$. We also show that
\end{abstract}

$$
\text { per } A(a \mid c)+\operatorname{per} A(b \mid c)+\operatorname{per} A(a \mid d)+\operatorname{per} A(b \mid d) \equiv 0 \bmod 4
$$

for all $a, b, c, d$, when $A$ is an integer matrix of odd order with all row and columns sums equal to $k \equiv 2 \bmod 4$.

\section{INTRODUCTION}

A Latin square is an $n \times n$ matrix consisting of $n$ distinct symbols where each symbol appears exactly once in each row and each column. Our Latin squares will have their rows and columns indexed by $[n]=\{1,2, \ldots, n\}$ and will also have their symbols chosen from $[n]$. Latin squares can then be thought of as a set of entries $\{(r, c, s)\} \subset$ $[n]^{3}$ where each distinct pair of entries agree in at most one coordinate. The three coordinates of an entry are its row index, column index and symbol. A diagonal of a Latin square is a selection of $n$ entries, with exactly one entry from each row and each column. The weight of a diagonal is the number of distinct symbols on that diagonal. A diagonal of weight $n$ is called a transversal. Historically, transversals in Latin squares were first used as the building blocks of mutually orthogonal Latin squares (MOLS). They have since garnered a lot of interest on their own (see [26] for a survey). A partial transversal of length $k$ is a selection of $k$ entries so that no two entries share the same row, column or symbol. A partial transversal of length $k<n$

Manuscript received 23rd February 2019, revised 10th July 2019, accepted 25th November 2019.

KEYWORDS. parity, Latin square, transversal, permanent, Latin rectangle, perfect matching, permanental minor, bipartite graph.

ACKNowledgements. Research supported by Endeavour Postgraduate Scholarship and NSERC CGS-D and by Australian Research Council grant DP150100506. 
is not the same thing as a diagonal of weight $k$. While these objects are related, the distinction is important when counting them.

Over half a century ago, Ryser [22] put forward the following famous conjecture.

Conjecture 1.1 (Ryser's Conjecture). Every Latin square of odd order has a transversal.

This conjecture has been shown to be true for $n \leqslant 9$ by computation [18]. In 1990, Balasubramanian [6] showed that the number of transversals in a Latin square of even order is itself even. He claimed that this was a partial proof of a stronger form of Conjecture 1.1, namely that the number of transversals in a Latin square of order $n$ should agree with $n$ mod 2. Despite [6] attributing this conjecture to [22] it is nowhere to be found in the latter work. It is possible that Ryser made the conjecture, but we have been unable to find evidence of this. It is also worth remarking that many Latin squares of odd order have an even number of transversals, so the stronger form of Conjecture 1.1 is false. However, it does raise the intriguing possibility of proving existence of objects (in this case, transversals) by studying congruences satisfied by the number of those objects. We achieve this on a very modest scale (cf. Example 3.14), but mostly use it as motivation to unearth what we consider to be interesting patterns in numbers of transversals and related quantities.

Akbari and Alipour [2] developed the ideas from Balasubramanian's result to show that the number of diagonals with weight $n-1$ is even in every Latin square. We outline and expand on these ideas in $\S 2$. We then show that the number of transversals in a Latin square of order $n \equiv 2 \bmod 4$ is necessarily a multiple of 4 . We show this by exploiting a notion of parity for transversals. Parity of permutations is, of course, a very well-known concept. To our knowledge it had not previously been usefully applied to transversals. However, applying it to the permutations that define the rows, columns and symbols of a single Latin square or set of MOLS has previously revealed many insights $[1,3,4,5,9,10,11,12,13,14,15,17,23,25]$. In particular, each Latin square has a row-parity $\pi_{r}$, which is the $\mathbb{Z}_{2}$ sum of the parities of the permutations that define the rows, and a column parity $\pi_{c}$ which is defined similarly for columns (there is also a symbol parity $\pi_{s}$, but it is a function of $\pi_{r}$ and $\pi_{c}$, see $[10,14,25]$ ). We will demonstrate several new ways to partition Latin squares of certain orders into two types (independently of their $\pi_{r}$ and $\pi_{c}$ ).

In $\S 3$, we consider counts of transversals in (not necessarily square) submatrices of Latin squares. A Latin array is a matrix of symbols in which no symbol is repeated within any row, or within any column. A transversal of an $m \times n$ Latin array is a selection of $\min (m, n)$ entries in which no pair of entries share their row, column or symbol. Transversals in Latin arrays are naturally encountered in attempts to find transversals of Latin squares by induction. They have been the subject of a recent burst of activity $[7,8,16,19]$ on the question posed in [2] of how many symbols in a Latin array are enough to make a transversal unavoidable. We take a different tack, considering congruences satisfied by the number of transversals in Latin arrays formed by removing one row and/or one column from a Latin square.

Transversals are diagonals with the maximum possible number of symbols. In $\S 4$ we count diagonals according to how many symbols they contain and demonstrate several relationships between the resulting numbers. In doing so we extend on results obtained in $[2,6]$.

One of the key tools in our results is a matrix function known as the permanent. Let $M_{n}(\mathbb{Z})$ denote the $n \times n$ integer matrices. The permanent of a matrix $A=\left[a_{i j}\right]$ 
in $M_{n}(\mathbb{Z})$ is defined by

$$
\operatorname{per} A=\sum_{\sigma \in \mathcal{S}_{n}} \prod_{i=1}^{n} a_{i \sigma(i)}
$$

where the sum is over all permutations in the symmetric group $\mathcal{S}_{n}$ on $[n]$. At several points, we use Ryser's formula [21] to compute the permanent of a matrix. It states that for $A=\left[a_{i j}\right] \in M_{n}(\mathbb{Z})$,

$$
\operatorname{per} A=\sum_{S \subseteq[n]}(-1)^{n-|S|} \prod_{i=1}^{n} \sum_{j \in S} a_{i j} .
$$

We also make frequent use of the fact that (1) agrees, modulo 2, with the definition of the determinant. In a determinant, some diagonals are given a negative sign, but $-1 \equiv 1 \bmod 2$ so per $A \equiv \operatorname{det} A \bmod 2$. As a simple example of how this observation can be used, we have:

LEMma 1.2. If $A \in M_{n}(\mathbb{Z})$ is such that all row sums are even, then $\operatorname{det} A$ and per $A$ are both even.

Proof. Since the sum of the entries in each row is even, the columns of $A$ are linearly dependent over $\mathbb{Z}_{2}$. Thus, $\operatorname{det} A \equiv 0 \bmod 2$, from which the claim follows.

Throughout the paper, $J$ is an all-ones matrix of the appropriate order and $\Lambda_{n}^{k}$ is the set of all $(0,1)$-matrices of order $n$ which contain exactly $k$ ones in each row and each column. We also need notation for the conjugates of a Latin square $L$. For each permutation $a b c$ in $\mathcal{S}_{3}$ (written in image notation) there is an $a b c$-conjugate of $L$. It is the Latin square obtained by applying the permutation $a b c$ to the three coordinates in the entries of $L$. For example, the 213-conjugate of $L$ is the usual matrix transpose of $L$.

\section{Transversals of Latin squares of EVEn order}

In this section, we layout the ideas used first by Balasubramanian [6], then again by Akbari and Alipour [2] to count the number of transversals in even ordered Latin squares modulo 2. For consistency with [2], we will define $E_{m}=E_{m}(L)$ to be the number of diagonals in $L$ that contain exactly $m$ distinct symbols. In particular, $E_{n}(L)$ is the number of transversals in $L$ if $L$ has order $n$. The key idea is to count transversals using inclusion-exclusion.

DEFINITION 2.1. Let $f\left(x_{1}, \ldots, x_{n}\right)$ be an arbitrary polynomial from $\mathbb{R}^{n}$ to $\mathbb{R}$. Then $\langle r\rangle f$ denotes the sum of the values of $f$ at the $\left(\begin{array}{l}n \\ r\end{array}\right)$ vectors in $\mathbb{R}^{n}$ which have $r$ coordinates equal to 1 and $n-r$ coordinates equal to 0 .

The following result is a slight generalisation of both [6, Lemma 2] and [2, Theorem 2.1].

LEMma 2.2. Let $f\left(x_{1}, \ldots, x_{n}\right)$ be an arbitrary polynomial from $\mathbb{R}^{n}$ to $\mathbb{R}$. Then the sum of the coefficients of monomials in $f$ containing exactly $m$ distinct variables is

$$
\sum_{r=0}^{m}(-1)^{m-r}\left(\begin{array}{c}
n-r \\
n-m
\end{array}\right)\langle r\rangle f .
$$


Proof. Suppose that $\mu$ is a monic monomial containing $t$ distinct variables. Then

$$
\begin{aligned}
\sum_{r=0}^{m}(-1)^{m-r}\left(\begin{array}{c}
n-r \\
n-m
\end{array}\right)\langle r\rangle \mu & =\sum_{r=t}^{m}(-1)^{m-r}\left(\begin{array}{c}
n-r \\
n-m
\end{array}\right)\left(\begin{array}{c}
n-t \\
r-t
\end{array}\right) \\
& =\left(\begin{array}{c}
n-t \\
n-m
\end{array}\right) \sum_{r=t}^{m}(-1)^{m-r}\left(\begin{array}{c}
m-t \\
r-t
\end{array}\right) \\
& = \begin{cases}1 & \text { if } t=m, \\
0 & \text { otherwise. }\end{cases}
\end{aligned}
$$

The result follows.

For any transversal, $\left\{\left(r_{i}, c_{i}, s_{i}\right)\right\}$, we define three corresponding permutations of the index set $[n]$ by $\sigma_{r}\left(r_{i}\right)=c_{i}, \sigma_{c}\left(c_{i}\right)=s_{i}$ and $\sigma_{s}\left(s_{i}\right)=r_{i}$. The following result is immediate.

Lemma 2.3. Let $T=\left\{\left(r_{i}, c_{i}, s_{i}\right)\right\}$ be a transversal of a Latin square $L$ with corresponding permutations $\sigma_{r}, \sigma_{c}$ and $\sigma_{s}$. Then $\sigma_{r} \circ \sigma_{c} \circ \sigma_{s}$ is the identity permutation.

Proof. For any given symbol $s_{i}$, we have $s_{i} \stackrel{\sigma_{s}}{\longrightarrow} r_{i} \stackrel{\sigma_{r}}{\longrightarrow} c_{i} \stackrel{\sigma_{c}}{\longrightarrow} s_{i}$.

As an immediate corollary of Lemma 2.3, we have that $\varepsilon\left(\sigma_{r}\right)+\varepsilon\left(\sigma_{c}\right)+\varepsilon\left(\sigma_{s}\right)=0$, where $\varepsilon: \mathcal{S}_{n} \rightarrow \mathbb{Z}_{2}$ is the standard parity homomorphism on the symmetric group $\mathcal{S}_{n}$. Thus, we can classify transversals into four types: $T^{000}, T^{011}, T^{101}$ or $T^{110}$ where the superscript records the parities of $\sigma_{r}, \sigma_{c}$ and $\sigma_{s}$, respectively. We will use these parities to aid in counting transversals.

Definition 2.4. Let $L$ be a Latin square of order $n$. The parity of a transversal is the parity of the permutation $\sigma_{r}$ defined above. We define $E_{n}^{ \pm}(L)$ to be the number of transversals in $L$ with $\varepsilon\left(\sigma_{r}\right)=0$ (even transversals) minus the number of transversals in $L$ with $\varepsilon\left(\sigma_{r}\right)=1$ (odd transversals).

Though the symbols in our Latin square $L$ are normally from $[n]$, we sometimes need to utilise the corresponding matrix $L[X]$, where each symbol $i$ is replaced with a variable $x_{i}$.

THEOREM 2.5. Let $L$ be a Latin square of order $n$. Then

$$
E_{n}(L)=\sum_{r=0}^{n}(-1)^{n-r}\langle r\rangle \text { per } L[X],
$$

and

$$
E_{n}^{ \pm}(L)=\sum_{r=0}^{n}(-1)^{n-r}\langle r\rangle \operatorname{det} L[X] .
$$

Proof. We use the $m=n$ case of Lemma 2.2. The terms in per $L[X]$ which include $n$ distinct variables correspond to the transversals in $L$. Similar terms in $\operatorname{det} L[X]$ correspond to the transversals of $L$ up to sign. Any transversal which has even parity increases the sum in (4) by 1 and any odd transversal decreases the sum by 1 .

The following lemma is adapted from [20, Lemma 1].

Lemma 2.6. Let $A=\left[a_{i j}\right]$ be a $(0,1)$-matrix of even order. Define $A^{*}=\left[b_{i j}\right]$ by

$$
b_{i j}= \begin{cases}a_{i j} & \text { if the } i^{\text {th }} \text { row has an even number of ones, } \\ 1-a_{i j} & \text { otherwise. }\end{cases}
$$

Then $\operatorname{det} A+\operatorname{det} A^{*}$ is even. 
Proof. Let $\delta$ be a row vector of ones. By permuting rows if necessary, we may assume that the first $k$ rows of $A$ have odd sum and the remaining rows have even sum (permuting rows may alter the sign of the determinant, but this does not matter modulo 2). Thus, we have

$$
\begin{gathered}
\pm \operatorname{det} A^{*}=\operatorname{det}\left(\begin{array}{c}
\delta-A_{1} \\
\delta-A_{2} \\
\vdots \\
\delta-A_{k} \\
A_{k+1} \\
\vdots \\
A_{n}
\end{array}\right)=\operatorname{det}\left(\begin{array}{c}
\delta-A_{1} \\
A_{1}-A_{2} \\
\vdots \\
A_{1}-A_{k} \\
A_{k+1} \\
\vdots \\
A_{n}
\end{array}\right)=\operatorname{det}\left(\begin{array}{c}
-A_{1} \\
A_{1}-A_{2} \\
\vdots \\
A_{1}-A_{k} \\
A_{k+1} \\
\vdots \\
A_{n}
\end{array}\right)+\operatorname{det}\left(\begin{array}{c}
\delta \\
A_{1}-A_{2} \\
\vdots \\
A_{1}-A_{2} \\
\vdots \\
A_{k+1}-A_{k} \\
A_{k+1} \\
\vdots \\
A_{n}
\end{array}\right) \equiv \operatorname{det} A \bmod 2 \\
\\
=(-1)^{k} \operatorname{det} A+\operatorname{det}\left(\begin{array}{c} 
\\
\end{array}\right)
\end{gathered}
$$

by Lemma 1.2. The result follows.

Consider the special case of Lemma 2.6 where the row sums of $A$ are all the same. If all of the row sums are even, then the result shows nothing interesting. However, when each row sum is odd, Lemma 2.6 tells us that $\operatorname{det} A+\operatorname{det}(J-A) \equiv$ $0 \bmod 2$. Balasubramanian [6] used this result and (3) to show the following theorem (actually, [6] showed a generalisation of this result, which we discuss later). We give a full proof here, as we will use a similar technique for several of our new results.

THEOREM 2.7. If $L$ is a Latin square of even order $n$ then $L$ has an even number of transversals.

Proof. Note that $E_{n}(L) \equiv E_{n}^{ \pm}(L) \bmod 2$. We pair up complementary terms in (4). In other words, each term of the sum $\langle r\rangle \operatorname{det} L[X]$ is paired with the unique term in $\langle n-r\rangle \operatorname{det} L[X]$ for which the indexing zero-one vectors sum to the all-ones vector. For each of these pairs of terms, we have one of two situations. If $r$ is even, then $n-r$ is also even and so both determinants are even, by Lemma 1.2. Alternatively, if $r$ is odd, then each row sum in $L[X]$ is odd, so $\operatorname{det} L[X]+\operatorname{det}(J-L[X]) \equiv 0 \bmod 2$ by Lemma 2.6. Thus, each of the $2^{n-1}$ pairs contributes a multiple of two to the summation in (4). The result follows.

To proceed, we need a few linear algebraic results.

LEMMA 2.8. If $A \in \Lambda_{n}^{k}$ where both $n$ and $k$ are even, then $\operatorname{det} A \equiv 0 \bmod 4$.

Proof. Since $A \in \Lambda_{n}^{k}$, we have that $\operatorname{det} A$ is a multiple of $k \cdot \operatorname{gcd}(n, k)$, by [20, Theorem 2]. The desired result follows, since $k$ and $\operatorname{gcd}(n, k)$ are both even.

LEMma 2.9. Let $n \equiv 2 \bmod 4$ and $k \equiv 1 \bmod 2$. If $A \in \Lambda_{n}^{k}$, then

$$
\operatorname{det} A+\operatorname{det}(J-A) \equiv 0 \bmod 4 \text {. }
$$

Proof. Since $A \in \Lambda_{n}^{k}$, we have that

$$
k \operatorname{det}(J-A)=(-1)^{n-1}(n-k) \operatorname{det} A,
$$


by [20, Lemma 1$]$. The result follows by noting that $n-k \equiv k \bmod 4$ and that $k$ has a multiplicative inverse modulo 4 .

We now have the framework to start counting transversals. The proof of our next result is very similar to that of Theorem 2.7.

THEOREM 2.10. If $L$ is a Latin square of order $n \equiv 2 \bmod 4$ then $E_{n}^{ \pm}(L) \equiv 0 \bmod 4$.

Proof. Again, we pair up complementary terms in (4). That is, each term of the sum $\langle r\rangle \operatorname{det} L[X]$ is paired with the unique term in $\langle n-r\rangle \operatorname{det} L[X]$ for which the indexing zero-one vectors sum to the all-ones vector. For each of these pairs of terms, we have one of two situations. If $r$ is even, then $n-r$ is also even and we use Lemma 2.8 twice to show that both terms are a multiple of four. Alternatively, if $r$ is odd, then we use Lemma 2.9 to show that the terms sum to a multiple of four. The result follows.

We now use Theorem 2.10 to show our first main result, which strengthens Theorem 2.7 for Latin squares of singly-even order.

Theorem 2.11. If $L$ is a Latin square of order $n \equiv 2 \bmod 4$ then $E_{n}(L) \equiv 0 \bmod 4$.

Proof. Let $T$ be the set of transversals of $L$. We define $w, x, y$ and $z$ to be the number of transversals of type $T^{000}, T^{011}, T^{101}$ and $T^{110}$, respectively. By definition, we have

$$
w+x+y+z=E_{n}(L)
$$

and

$$
w+x-y-z=E_{n}^{ \pm}(L) .
$$

Let $L^{\prime}$ be the 312-conjugate of $L$. There is a natural bijection between $T$ and the set of transversals of $L^{\prime}$. Each transversal in $L^{\prime}$ must be of the form $\left\{\left(c_{i}, s_{i}, r_{i}\right)\right\}$, where $\left\{\left(r_{i}, c_{i}, s_{i}\right)\right\} \in T$. The parity of each transversal in $L^{\prime}$ depends on $\sigma_{c}$ for the corresponding transversal in $T$, so we have

$$
w-x+y-z=E_{n}^{ \pm}\left(L^{\prime}\right) .
$$

Similarly, if $L^{\prime \prime}$ is the 231-conjugate of $L$, then each transversal of $L^{\prime \prime}$ has parity matching that of $\sigma_{s}$ for the corresponding transversal in $T$. So we have

$$
w-x-y+z=E_{n}^{ \pm}\left(L^{\prime \prime}\right) .
$$

The sum of (6), (7), (8) and (9) gives us

$$
4 w=E_{n}(L)+E_{n}^{ \pm}(L)+E_{n}^{ \pm}\left(L^{\prime}\right)+E_{n}^{ \pm}\left(L^{\prime \prime}\right) .
$$

Theorem 2.10 applied to $L, L^{\prime}$ and $L^{\prime \prime}$ tells us that $E_{n}(L) \equiv 0 \bmod 4$.

Based on computation of small squares, it seems that Theorem 2.7 and Theorem 2.11 are the only general modular restrictions on the number of transversals of a Latin square. By considering sets of Latin squares that are connected by turning intercalates (that is, replacing a subsquare $\left[\begin{array}{ll}a & b \\ b & a\end{array}\right]$ with $\left[\begin{array}{ll}b & a \\ a & b\end{array}\right]$ ), we were able to find Latin squares that satisfy every other congruence with small modulus. For example, suppose that $9 \leqslant n \leqslant 11$ and $0 \leqslant k<m \leqslant 32$. Except where it would violate Theorem 2.7 or 
Theorem 2.11, there is some subset of intercalates in these Latin squares

\begin{tabular}{|l|l|l|l|l|l|l|l|l|}
\hline 1 & 2 & 3 & 4 & 5 & 6 & 7 & 8 & 9 \\
\hline 2 & 1 & 4 & 3 & 6 & 5 & 9 & 7 & 8 \\
\hline 3 & 6 & 1 & 8 & 7 & 9 & 5 & 2 & 4 \\
\hline 4 & 3 & 5 & 6 & 9 & 7 & 8 & 1 & 2 \\
\hline 5 & 4 & 2 & 9 & 8 & 1 & 6 & 3 & 7 \\
\hline 6 & 9 & 7 & 5 & 3 & 8 & 2 & 4 & 1 \\
\hline 7 & 8 & 9 & 1 & 2 & 3 & 4 & 5 & 6 \\
\hline 8 & 5 & 6 & 7 & 4 & 2 & 1 & 9 & 3 \\
\hline 9 & 7 & 8 & 2 & 1 & 4 & 3 & 6 & 5 \\
\hline
\end{tabular}

\begin{tabular}{|c|c|c|c|c|c|c|c|c|c|}
\hline 1 & 2 & 3 & 4 & 5 & 6 & 7 & 8 & 9 & 10 \\
\hline 2 & 1 & 4 & 3 & 6 & 5 & 8 & 7 & 10 & 9 \\
\hline 3 & 6 & 5 & 7 & 2 & 8 & 10 & 9 & 4 & 1 \\
\hline 4 & 5 & 6 & 8 & 7 & 9 & 2 & 10 & 1 & 3 \\
\hline 5 & 8 & 7 & 9 & 1 & 10 & 4 & 3 & 2 & 6 \\
\hline 6 & 4 & 8 & 10 & 9 & 7 & 1 & 2 & 3 & 5 \\
\hline 7 & 3 & 10 & 5 & 8 & 1 & 9 & 4 & 6 & 2 \\
\hline 8 & 7 & 9 & 6 & 10 & 2 & 3 & 1 & 5 & 4 \\
\hline 9 & 10 & 1 & 2 & 3 & 4 & 5 & 6 & 7 & 8 \\
\hline 10 & 9 & 2 & 1 & 4 & 3 & 6 & 5 & 8 & 7 \\
\hline
\end{tabular}

\begin{tabular}{|c|c|c|c|c|c|c|c|c|c|c|}
\hline 1 & 2 & 3 & 4 & 5 & 6 & 7 & 8 & 9 & 10 & 11 \\
\hline 2 & 1 & 4 & 3 & 6 & 5 & 8 & 7 & 11 & 9 & 10 \\
\hline 3 & 8 & 1 & 6 & 7 & 10 & 11 & 9 & 4 & 5 & 2 \\
\hline 4 & 11 & 2 & 8 & 9 & 7 & 5 & 10 & 1 & 3 & 6 \\
\hline 5 & 3 & 6 & 10 & 8 & 9 & 1 & 2 & 7 & 11 & 4 \\
\hline 6 & 4 & 7 & 9 & 10 & 11 & 2 & 3 & 8 & 1 & 5 \\
\hline 7 & 5 & 8 & 11 & 4 & 2 & 10 & 1 & 3 & 6 & 9 \\
\hline 8 & 7 & 9 & 5 & 11 & 1 & 6 & 4 & 10 & 2 & 3 \\
\hline 9 & 10 & 11 & 1 & 2 & 3 & 4 & 5 & 6 & 7 & 8 \\
\hline 10 & 6 & 5 & 7 & 3 & 8 & 9 & 11 & 2 & 4 & 1 \\
\hline 11 & 9 & 10 & 2 & 1 & 4 & 3 & 6 & 5 & 8 & 7 \\
\hline
\end{tabular}

that can be turned to give a Latin square of order $n$ with $k \bmod m$ transversals. We also found examples for $12 \leqslant n \leqslant 16$ with the same property, but we do not display them here for the sake of space. For $n \leqslant 7$, there are some sporadic values of $k, m \leqslant 16$ where no Latin square of order $n$ contains $k \bmod m$ transversals. For $n=8$, there is no Latin square that contains $22 \bmod 63$ transversals, while there exists a Latin square that contains $k$ mod $m$ transversals for all other $0 \leqslant k<m \leqslant 64$ that satisfy Theorem 2.7. However, we believe that the restrictions for $n \leqslant 8$ are not interesting; they are simply a result of there being comparatively few Latin squares of these orders.

The proof of Theorem 2.11 leads us to the following interesting property.

COROllary 2.12. Let $L$ be a Latin square of order $n \equiv 2 \bmod 4$. The numbers of transversals in $L$ of types $T^{000}, T^{110}, T^{101}$ and $T^{110}$ are all equal modulo 2.

Proof. Define $w, x, y$ and $z$ as in Theorem 2.11. Adding (6) to (7) we find that $2 w+2 x=E_{n}(L)+E_{n}^{ \pm}(L) \equiv 0 \bmod 4$ (by Theorem 2.10 and Theorem 2.11) which gives us that $w \equiv x \bmod 2$. Similarly, $(6)+(8)$ and $(6)+(9)$ tell us that $w \equiv y \bmod 2$ and $w \equiv z \bmod 2$, respectively.

It is important to remark that Theorem 2.11 is less general in one respect than Balasubramanian's Theorem [6]. Balasubramanian proved that the number of transversals in any row-Latin square of even order is even (a row-Latin square of order $n$ is an $n \times n$ matrix in which each row is a permutation of $[n])$. Theorem 2.11 does not generalise to row-Latin squares. Below we give two row-Latin squares whose number of 
transversals is not a multiple of 4 . The row-Latin square of order 2 has 2 transversals and the row-Latin square of order 6 has 6 transversals.

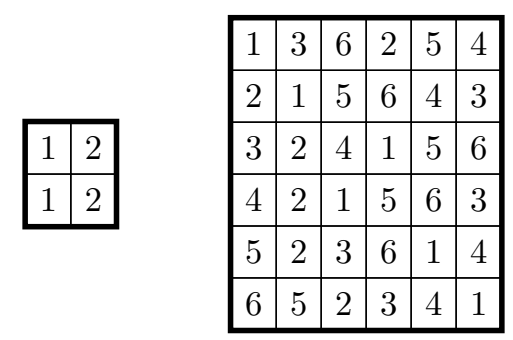

Computational evidence suggests the following generalisation of Theorem 2.11 and Corollary 2.12.

Conjecture 2.13. Let $L$ be a Latin square of even order $n$. Let $w, x, y$ and $z$ be the number of transversals in $L$ of types $T^{000}, T^{011}, T^{101}$ and $T^{110}$, respectively. Then

(a) $E_{n}(L) \equiv E_{n}^{ \pm}(L) \bmod 4$ and

(b) $w \equiv x \equiv y \equiv z \bmod 2$.

Conjecture 2.13 is true for $n \equiv 2 \bmod 4$ since $E_{n}(L) \equiv E_{n}^{ \pm}(L) \equiv 0 \bmod 4$ (by Theorem 2.10 and Theorem 2.11) and $w \equiv x \equiv y \equiv z \bmod 2$ (by Corollary 2.12). Note that there are many Latin squares of odd order for which Conjecture 2.13 is not true.

Lemma 2.14. The conditions (a) and (b) in Conjecture 2.13 are equivalent for Latin squares of even order.

Proof. By Theorem 2.7, we know that $E_{n}(L) \equiv 0 \bmod 2$ when $n$ is even, showing condition (a) is equivalent to $E_{n}(L)+E_{n}^{ \pm}(L) \equiv 0 \bmod 4$. We may use the same idea as the proof of Corollary 2.12 to show the result.

\section{Transversals of Depleted Latin SQUares}

In this section we give a number of results around the common theme of transversals of depleted Latin squares, that is, matrices formed by removing a row and/or a column of a Latin square. This depleted Latin square is a Latin array.

Given an $n \times n$ matrix $A$, we use $\nu(A)$ to denote the $\mathbb{Z}_{2}$-nullity of $A$ and we use $A(i \mid j)$ to denote the $(n-1) \times(n-1)$ matrix obtained by deleting row $i$ and column $j$ from $A$. We start by considering the permanent of this submatrix, which is analogous to the consideration of minors when computing the determinant.

ThEOREM 3.1. Let $A \in M_{n}(\mathbb{Z})$ for $n>1$. Then

- $\operatorname{per} A(i \mid j) \equiv 0 \bmod 2$ for all $i, j$ if and only if $\nu(A) \geqslant 2$.

- $\operatorname{per} A(i \mid j) \equiv 1 \bmod 2$ for all $i, j$ if and only if $\nu(A)=1$ and all row and column totals of $A$ are even.

Proof. It suffices to show analogous properties for determinants since the determinant and permanent agree modulo 2 . All calculations in this proof will be working over $\mathbb{Z}_{2}$, and all minors will be of order $n-1$.

If $\nu(A) \geqslant 2$, then for all $i, j$ we know that $\nu(A(i \mid j)) \geqslant 1$ so $\operatorname{det} A(i \mid j) \equiv 0 \bmod 2$.

If $\nu(A)=0$, then $A$ has an inverse so the adjugate $\operatorname{adj}(A)$ has full rank and hence is not a multiple of $J$ (given that $n>1$ ). Hence not all minors of $A$ are equal.

So suppose that $\nu(A)=1$, and hence $\operatorname{det} A=0$. Since $\nu(A)=1$ there is at least one minor of $A$ that equals 1 . 
If there is any row or column of $A$ with odd sum, then expanding the determinant in that row/column shows that $A$ has at least one minor which is zero, and hence not all minors are equal.

It remains to treat the case where each row and column sum of $A$ is even. It suffices to show $\operatorname{det} A(1 \mid 1) \equiv \operatorname{det} A(2 \mid 1) \bmod 2$. But

$$
\operatorname{det} A(1 \mid 1)+\operatorname{det} A(2 \mid 1)=\operatorname{det}\left(\begin{array}{cccc}
a_{12}+a_{22} & a_{13}+a_{23} & \cdots & a_{1 n}+a_{2 n} \\
a_{32} & a_{33} & \cdots & a_{3 n} \\
a_{42} & a_{43} & \cdots & a_{4 n} \\
\vdots & \vdots & \ddots & \vdots \\
a_{n 2} & a_{n 3} & \cdots & a_{n n}
\end{array}\right) \equiv 0 \bmod 2
$$

since all column sums are even (cf. Lemma 1.2).

Since $\nu(A) \geqslant 1$ whenever all row totals are even, we have:

COROllary 3.2. Let $A \in M_{n}(\mathbb{Z})$ be such that all row and column sums are even. Then per $A(a \mid c) \equiv \operatorname{per} A(b \mid d) \bmod 2$ for all $a, b, c, d$.

The previous result gave a congruence mod 2. Our next results involve congruences $\bmod 4$.

Theorem 3.3. Suppose that $n$ is odd and $k \equiv 2 \bmod 4$. If $A \in \Lambda_{n}^{k}$, then

$$
\text { per } A(a \mid c)+\operatorname{per} A(b \mid c)+\operatorname{per} A(a \mid d)+\operatorname{per} A(b \mid d) \equiv 0 \bmod 4
$$

for any $a, b, c, d$.

Proof. If $a=b($ or symmetrically, $c=d)$, then $2(\operatorname{per} A(a \mid c)+\operatorname{per} A(a \mid d)) \equiv 0 \bmod 4$, by Corollary 3.2. Hence, it suffices to consider the case when $a=c=1$ and $b=d=2$. Define

$$
B=\left(\begin{array}{cccc}
a_{11}+a_{21}+a_{12}+a_{22} & a_{13}+a_{23} & \cdots & a_{1 n}+a_{2 n} \\
a_{31}+a_{32} & a_{33} & \cdots & a_{3 n} \\
a_{41}+a_{42} & a_{43} & \cdots & a_{4 n} \\
\vdots & \vdots & \ddots & \vdots \\
a_{n 1}+a_{n 2} & a_{n 3} & \cdots & a_{n n}
\end{array}\right)
$$

Note that $B$ has order $n-1$ and that its first row and column each sum to $2 k \equiv$ $0 \bmod 4$, while its other rows and columns each sum to $k$. Also, by multilinearity of the permanent,

$$
\begin{array}{r}
\operatorname{per} B=\operatorname{per}\left(\begin{array}{cccc}
a_{12}+a_{22} & a_{13}+a_{23} & \cdots & a_{1 n}+a_{2 n} \\
a_{32} & a_{33} & \cdots & a_{3 n} \\
a_{42} & a_{43} & \cdots & a_{4 n} \\
\vdots & \vdots & \ddots & \vdots \\
a_{n 2} & a_{n 3} & \cdots & a_{n n}
\end{array}\right) \\
+\operatorname{per}\left(\begin{array}{cccc}
a_{11}+a_{21} & a_{13}+a_{23} & \cdots & a_{1 n}+a_{2 n} \\
a_{31} & a_{33} & \cdots & a_{3 n} \\
a_{41} & a_{43} & \cdots & a_{4 n} \\
\vdots & \vdots & \ddots & \vdots \\
a_{n 1} & a_{n 3} & \cdots & a_{n n}
\end{array}\right) \\
=\operatorname{per} A(1 \mid 1)+\operatorname{per} A(2 \mid 1)+\operatorname{per} A(1 \mid 2)+\operatorname{per} A(2 \mid 2) .
\end{array}
$$


Next, apply (2) to calculate per $B$ :

$$
\operatorname{per} B=\sum_{S \subseteq[n-1]}(-1)^{n-1-|S|} \prod_{i=1}^{n-1} \sum_{j \in S} b_{i j} .
$$

Fix a set $S_{0}$ and consider the terms corresponding to $S_{0}$ and its complement in the outer summation. We have,

$$
\begin{aligned}
(-1)^{n-1-\left|S_{0}\right|} \prod_{i=1}^{n-1} \sum_{j \in S_{0}} b_{i j}+(-1)^{\left|S_{0}\right|} \prod_{i=1}^{n-1} \sum_{j \notin S_{0}} b_{i j} \\
=(-1)^{\left|S_{0}\right|}\left(\prod_{i=1}^{n-1} x_{i}+\left(2 k-x_{1}\right) \prod_{i=2}^{n-1}\left(k-x_{i}\right)\right) \\
\equiv(-1)^{\left|S_{0}\right|}\left(2 \prod_{i=1}^{n-1} x_{i}-k \sum_{j=2}^{n-1} \prod_{i \neq j} x_{i}\right) \bmod 4
\end{aligned}
$$

where

$$
x_{i}=\sum_{j \in S_{0}} b_{i j} .
$$

Now $\sum_{i} x_{i}$ is even, so there is an even number of choices of $i$ for which $x_{i}$ is even. If this number is non-zero, then (11) is clearly 0 modulo 4 . So we may assume that every $x_{i}$ is odd. But then (11) is 0 modulo 4 again, since each term in the sum is odd and there is an odd number of summands.

Theorem 3.4. Let $n \equiv 1 \bmod 2$ and $k \equiv 2 \bmod 4$. If $A \in \Lambda_{n}^{k}$, then

$$
\operatorname{per} A+2 \operatorname{per}(J-A) \equiv 0 \bmod 4 .
$$

Proof. By inclusion-exclusion,

$$
\operatorname{per}(J-A)=\sum_{i=0}^{n}(-1)^{i}(n-i) ! \tau_{i}(A) \equiv \tau_{n-1}(A)-\operatorname{per} A \bmod 2,
$$

where $\tau_{i}(A)$ is the sum of the permanents of all $i \times i$ submatrices of $A$. However, per $A$ is even by Lemma 1.2 , so per $A+2 \operatorname{per}(J-A) \equiv \operatorname{per} A+2 \tau_{n-1}(A) \bmod 4$. Next, define an $n \times n$ matrix $C=\left[c_{i j}\right]$ by $c_{i j}=$ per $A(i \mid j)$. By Corollary 3.2 and Theorem 3.3, we know that modulo 4 each pair of rows of $C$ either agrees in every position or differs by 2 in every position. Hence, up to row and column permutations, $C$ has the block form

$$
\left(\begin{array}{ll}
C_{1} & C_{2} \\
C_{3} & C_{4}
\end{array}\right)
$$

where each entry in $C_{2} \cup C_{3}$ differs from each entry in $C_{1} \cup C_{4}$ by 2 mod 4 . Note that some blocks may be vacuous, but one of the four blocks must have odd dimensions. Without loss of generality, we choose it to be $C_{1}$. Now, partition $A$ into 4 blocks

$$
\left(\begin{array}{ll}
A_{1} & A_{2} \\
A_{3} & A_{4}
\end{array}\right)
$$

whose dimensions and locations match the corresponding block of $C$. Define $n_{r}$ to be the total of a row $r$ in block $A_{1}$. Next, consider calculating per $A$ mod 4 by taking an expansion along row $r$ :

$$
\text { per } A=\sum_{j=1}^{n} a_{r j} c_{r j} \equiv n_{r} c_{r 1}+\left(k-n_{r}\right)\left(c_{r 1}+2\right) \equiv 2 c_{r 1}-2 n_{r} \bmod 4 .
$$


The answer must be independent of $r$, which means that $n_{r} \bmod 2$ is constant. Analogous statements hold for row totals in each block. In particular, $A_{3}$, which has an even number of rows, must contain an even number of ones. But then $A_{1}$ must also contain an even number of ones, given that the column totals of $A$ are even. It follows that $n_{r}$ must be even, so per $A \equiv 2 c_{r 1} \bmod 4$. Now,

$$
\tau_{n-1}(A)=\sum_{i, j} c_{i j} \equiv n^{2} c_{r 1} \equiv c_{r 1} \bmod 2 .
$$

So per $A+2 \tau_{n-1}(A) \equiv 4 c_{r 1} \equiv 0 \bmod 4$ and we are done.

In our next major result, we show that a stronger form of Lemma 1.2 can be obtained under some circumstances.

THEOREM 3.5. Let $A \in M_{n}(\mathbb{Z})$ where $n$ is odd. If all row sums are multiples of 4 and all column sums are even, then per $A \equiv 0 \bmod 4$.

Proof. We compute the permanent via (2):

$$
\text { per } A=\sum_{S \subseteq[n]}(-1)^{n-|S|} \prod_{i=1}^{n} \sum_{j \in S} a_{i j} .
$$

Let $r_{i}$ be the sum of row $i$ and $c_{j}$ be the sum of column $j$ of $A$. Fix a set $S=S_{0}$ of odd cardinality and consider the contribution from $S_{0}$ and its complement. We have,

$$
\begin{aligned}
\prod_{i=1}^{n} \sum_{j \in S_{0}} a_{i j}-\prod_{i=1}^{n} \sum_{j \notin S_{0}} a_{i j} & =\prod_{i=1}^{n} \sum_{j \in S_{0}} a_{i j}-\prod_{i=1}^{n}\left(r_{i}-\sum_{j \in S_{0}} a_{i j}\right) \\
& \equiv 2 \prod_{i=1}^{n} \sum_{j \in S_{0}} a_{i j} \bmod 4 .
\end{aligned}
$$

Since each column of $A$ has an even total,

$$
\sum_{i=1}^{n} \sum_{j \in S_{0}} a_{i j}=\sum_{j \in S_{0}} c_{j} \equiv 0 \bmod 2 .
$$

Since $n$ is odd, $\sum_{j \in S_{0}} a_{i j}$ must be even for at least one value of $i$ in (13). Thus, the product of the partial row sums must be even and (12) must be a multiple of 4 . Summing over $S_{0}$, the result follows.

Corollary 3.6. Let $A \in \Lambda_{n}^{4 k}$ for integers $k, n$ with $n$ odd. Then per $A \equiv 0 \bmod 4$.

It is well-known that perfect matchings in bipartite graphs can be counted using the permanent of the bi-adjacency matrix of the graph. Corollary 3.6 says that the number of perfect matchings will be a multiple of 4 in any $4 k$-regular bipartite graph with an odd number of vertices in each class of the bipartition. Indeed, Theorem 3.5 says that the same conclusion can be reached under weaker hypotheses. It suffices for all vertices in one class to have even degree, and all vertices in the other class to have their degree divisible by 4 .

We define $t_{i j}(L)$ to be the number of transversals in the Latin array formed by deleting the $i$ th row and $j$ th column of $L$. When clear from context, the shorthand $t_{i j}$ is used.

TheOREM 3.7. Let $L$ be a row-Latin square of order $n$. Then for all $a, b, c$,

$$
t_{a b} \equiv t_{a c} \bmod 2 \text {. }
$$


Proof. Without loss of generality, we may assume that $n \geqslant 2, a=1, b=1, c=2$. Let $L[X]=\left[x_{i j}\right]$ and define

$$
L[X]^{\prime}=\left(\begin{array}{ccccc}
1 & 1 & 0 & \cdots & 0 \\
x_{21} & x_{22} & x_{23} & \cdots & x_{2 n} \\
\vdots & \vdots & \vdots & \ddots & \vdots \\
x_{n 1} & x_{n 2} & x_{n 3} & \cdots & x_{n n}
\end{array}\right)
$$

Then $t_{a b}+t_{a c}$ is the number of terms in per $L[X]^{\prime}$ which have exactly $n-1$ symbols. Thus, by Lemma 2.2,

$$
t_{a b}+t_{a c}=\sum_{r=0}^{n-1}(-1)^{n-1-r}(n-r)\langle r\rangle \operatorname{per} L[X]^{\prime} \equiv \sum_{r=1}^{n-1}(n-r)\langle r\rangle \operatorname{det} L[X]^{\prime} \bmod 2 .
$$

If $n$ is odd, then we have two subcases. If $r$ is even, then $\langle r\rangle \operatorname{det} L[X]^{\prime}$ is even, by Lemma 1.2. If $r$ is odd, then $n-r$ is even, and so each term in the summation is even.

If $n$ is even, then we use a trick similar to Theorem 2.10 by pairing up complementary terms. Our result follows from Lemma 1.2 and Lemma 2.6 when $r$ is even and $r$ is odd, respectively.

This immediately gives us a surprisingly simple result which lays the groundwork for the patterns found in the remainder of the section.

Corollary 3.8. Let $L$ be a Latin square of order $n$. Then for all $a, b, c, d$,

$$
t_{a b} \equiv t_{c d} \bmod 2 .
$$

Proof. Since $L$ is a row-Latin square, $t_{a b} \equiv t_{a d}$ mod 2 by Theorem 3.7. Moreover, since the transpose of $L$ is a row-Latin square, $t_{a d} \equiv t_{c d} \bmod 2$.

This simple observation leads to several patterns relating to deleting a row and a column of a Latin square.

COROLlaRY 3.9. Let $R$ be an $(n-1) \times n$ row-Latin rectangle, where $n$ is even. Then the number of transversals in $R$ is even.

Proof. Let $L$ be some row-Latin square formed by adding one row to $R$. By definition, the number of transversals in $R$ is

$$
t_{n 1}(L)+t_{n 2}(L)+\cdots+t_{n n}(L) .
$$

Each of these terms is congruent modulo 2 (by Theorem 3.7) and $n$ is even.

Each $(n-1) \times n$ Latin rectangle $R$ has a unique completion to a Latin square $L$, and each transversal of $R$ corresponds to a so-called near transversal of $L$. Corollary 3.9 does not generalise to odd orders, as there are some rectangles that have an even number of transversals and other rectangles that have an odd number of transversals. If any row is removed from the Cayley table of a cyclic group of odd order, the resulting Latin rectangle has an odd number of transversals. This can been seen by combining two well-known features of the cyclic group tables of odd order. Firstly each near transversal extends to a (unique) transversal, and secondly there are an odd number of transversals.

We define $N_{r}=N_{r}(L)$ to be the number of diagonals of weight $n-1$ in $L$ where the symbol that appears in row $r$ also appears in another row of the diagonal. The following two results follow directly from the definition of $t_{i j}$. 
LEMMA 3.10. Let $L$ be a Latin square of order $n$. Then for any row $r$,

$$
\sum_{c=1}^{n} t_{r c}=E_{n}+N_{r}
$$

Proof. Each transversal in the matrix formed by deleting row $r$ and column $c$ extends to either a transversal of $L$ or a diagonal of weight $n-1$ depending on which symbol is in the cell $(r, c)$.

LEMmA 3.11. Let $L$ be a Latin square of order $n$. Then

$$
\sum_{r=1}^{n} \sum_{c=1}^{n} t_{r c}=n E_{n}+2 E_{n-1} .
$$

Proof. Across the whole summation, each transversal of $L$ is counted $n$ times (once for each entry in the transversal) and each diagonal with weight $n-1$ is counted twice (once for each entry containing the duplicated symbol).

Our next main result has a curious feature, which we explain after proving the result.

THEOREM 3.12. Let $L$ be a Latin square of odd order $n$. Then for any $r$ and $c$,

$$
t_{r c} \equiv E_{n} \bmod 2 \text {. }
$$

Proof. Since $n$ is odd, Corollary 3.8 ensures that

$$
t_{r c} \equiv \sum_{i=1}^{n} \sum_{j=1}^{n} t_{i j} \bmod 2 .
$$

Then Lemma 3.11 gives $t_{r c} \equiv n E_{n}+2 E_{n-1} \equiv E_{n} \bmod 2$, as desired.

Corollary 3.13. Let $L$ be a Latin square of order $n$. Then $N_{r}$ is even for all rows $r$.

Proof. Each term in $\sum_{c=1}^{n} t_{r c}$ is the same modulo 2. If $n$ is even, this sum is even, whereas if $n$ is odd, the sum is equivalent to $t_{r 1}$ modulo 2 . In either case, the sum is equivalent to $E_{n}$ modulo 2 , by Theorem 2.7 and Theorem 3.12, respectively. The result now follows from Lemma 3.10.

An interesting feature of Theorem 3.12 lies in the fact that a transversal of $L$ can be inferred without locating one. In each of the other previous results, the number of diagonals with specific properties is of a similar form: congruent to 0 modulo $m$ for some $m$. Congruences like this cannot be used to show existence of transversals. However, Theorem 3.12 gives a slightly different approach. In particular, if $t_{r c} \equiv$ $1 \bmod 2$ for any row and column, then there must exist a transversal in $L$ even if none go through the cell $(r, c)$.

EXAMPLE 3.14. Consider $L_{5}$ :

$$
\begin{array}{|l|l|l|l|l|}
\hline 1 & 2 & 3 & 4 & 5 \\
\hline 2 & 1 & 4 & 5 & 3 \\
\hline 3 & 4 & 5 & 1 & 2 \\
\hline 4 & 5 & 2 & 3 & 1 \\
\hline 5 & 3 & 1 & 2 & 4 \\
\hline
\end{array}
$$


Every transversal in $L_{5}$ goes through the shaded entry. In particular, there are no transversals including the entry in the top left corner. However, the main diagonal is the sole transversal in $L_{5}(1 \mid 1)$, so $t_{11}=1$. Thus, we can use Theorem 3.12 to deduce that at least one transversal exists in $L_{5}$ without finding such a transversal.

We finish the discussion of $t_{i j}$ with a rather curious pattern found for small orders. It is very much in the spirit of Theorem 3.3 (but does not seem to follow directly from it).

Conjecture 3.15. Let $L$ be a Latin square of order $n$. Then $t_{a c}+t_{b c}+t_{a d}+t_{b d} \equiv$ 0 mod 4 for all $a, b, c, d$.

In light of Corollary 3.8, Conjecture 3.15 implies a very specific structure for the matrix $\left[t_{i j}\right]$. Each pair of rows either agrees modulo 4 or differs in every column by 2 modulo 4. A similar observation holds for columns.

\section{Counting diagonals By their number of Symbols}

In this section, we look at relationships between the $E_{i}=E_{i}(L)$, that is, the counts of diagonals of $L$ according to how many symbols they contain. We will also be interested in $R_{i}=R_{i}(L)$ which we define to be shorthand for $\langle i\rangle$ per $L[X]$. Note that $R_{0}=0$. The $R_{i}$ are related to the $E_{i}$ by

$$
E_{m}=\sum_{r=1}^{m}(-1)^{m-r}\left(\begin{array}{c}
n-r \\
n-m
\end{array}\right) R_{r}
$$

where $n$ is the order of $L$. This relationship was given explicitly in [2] and can easily be derived from Lemma 2.2 .

In several proofs we will encounter $d_{n}$, the number of derangements in $\mathcal{S}_{n}$. From the well-known recurrence $d_{n}=n d_{n-1}+(-1)^{n}$, we learn that

$$
d_{n} \equiv 1 \bmod 4 \text { when } n \text { is even. }
$$

The following proposition is a list of identities which are either immediate from the definition of a Latin square or are proved in [6].

LEMMA 4.1. Let $L$ be a Latin square of order $n$.
(a) $R_{1}=n$,
(b) $R_{n-1}=n d_{n}$,
(c) $R_{n}=n$ !,
(d) $R_{2 i}$ is even for each integer $i$,
(e) $R_{i}+R_{n-i}$ is even if $n$ is even, and
(f) $R_{n / 2}$ is even if $n$ is even.

Balasubramanian [6] used (d) and (e) to show Theorem 2.7, while Akbari and Alipour [2] showed the following two results.

TheOREM 4.2. If $L$ is a Latin square of order $n \equiv 2 \bmod 4$ then $E_{n-3}$ is even.

THEOREM 4.3. If $L$ is a Latin square of order $n$ then $E_{n-1}$ is even.

We start with patterns in Latin squares of odd order. We have two direct corollaries of earlier results.

Corollary 4.4. If $L$ is a Latin square of odd order then $R_{4 k} \equiv 0 \bmod 4$ for each integer $k$.

Proof. Simply apply Corollary 3.6 to each matrix in the sum that defines $R_{4 k}$. 
COROLlary 4.5. If $L$ is a Latin square of odd order $n$ and $k \equiv 2 \bmod 4$, then

$$
R_{k}+2 R_{n-k} \equiv 0 \bmod 4 .
$$

Proof. Apply Theorem 3.4 to each of the complementary pairs in $R_{k}$ and $R_{n-k}$.

In addition, we have:

THEOREM 4.6. If $L$ is a Latin square of odd order $n$ then $E_{i}$ is even whenever $i$ is even.

Proof. By (14) we have

$$
E_{i} \equiv \sum_{j=1}^{i}\left(\begin{array}{l}
n-j \\
n-i
\end{array}\right) R_{j} \bmod 2
$$

Now, $R_{j}$ is even for even $j$, by Lemma $4.1(\mathrm{~d})$. When $j$ is odd, $\left(\begin{array}{c}n-j \\ n-i\end{array}\right)$ is even by Lucas' Theorem, given that $n-j$ is even and $n-i$ is odd. The result follows.

We also have the following strengthening of Theorem 4.3 for odd orders:

THEOREM 4.7. If $L$ is a Latin square of odd order $n$ then $E_{n-1} \equiv 0 \bmod 4$.

Proof. We compute $E_{n-1}$ utilising (14). We pair up the complementary terms in this summation, $(n-r) R_{r}-r R_{n-r}$. Within each of these pairs, we assume that $r$ is even, by replacing $r$ by $n-r$ if necessary. We examine two cases. First, if $r \equiv 0 \bmod 4$, then the second term vanishes modulo 4 and $(n-r) R_{r} \equiv 0 \bmod 4$ by Corollary 4.4 Alternatively, if $r \equiv 2 \bmod 4$ then $R_{r}$ is even, by Lemma $4.1(\mathrm{~d})$, so $(n-r) R_{r} \equiv R_{r} \bmod$ 4. Thus, $(n-r) R_{r}-r R_{n-r} \equiv R_{r}+2 R_{n-r} \bmod 4$. We may now use Corollary 4.5. Each pair of complementary terms sums to a multiple of four, so the result follows.

We now shift our attention to Latin squares of even order, where the results are based on the global relationship between the different $R_{i}$ values in contrast with the local nature of Corollary 4.4 and Corollary 4.5.

THEOREM 4.8. If $L$ is a Latin square of even order $n>2$ then

$$
E_{1}+E_{3}+\cdots+E_{n-1} \equiv E_{2}+E_{4}+\cdots+E_{n} \equiv n \bmod 4 .
$$

Proof. By (14),

$$
\begin{aligned}
\sum_{m=1}^{n / 2} E_{2 m-1} & =\sum_{m=1}^{n / 2} \sum_{r=1}^{2 m-1}(-1)^{2 m-1-r}\left(\begin{array}{c}
n-r \\
n-2 m+1
\end{array}\right) R_{r} \\
& =\sum_{r=1}^{n-1}(-1)^{n-r-1} R_{r} \sum_{s=1}^{\lceil(n-r) / 2\rceil}\left(\begin{array}{c}
n-r \\
2 s-1
\end{array}\right) \\
& =\sum_{r=1}^{n-1}(-2)^{n-r-1} R_{r} \equiv R_{n-1}-2 R_{n-2} \equiv n d_{n}-0 \equiv n \bmod 4
\end{aligned}
$$

by Lemma 4.1 and (15). If $n \geqslant 4$ then $\sum_{i=1}^{n} E_{i}=n ! \equiv 0 \bmod 4$, and the second congruence follows.

COROLlary 4.9. Every Latin square has an even number of diagonals that contain an even number of symbols.

Proof. The order 2 case is trivial and Theorem 4.8 takes care of all larger even orders. The odd case is immediate from Theorem 4.6.

COROLlary 4.10. Every Latin square of order $n>1$ has an even number of diagonals that contain an odd number of symbols. 
Proof. There are $n ! \equiv 0 \bmod 2$ diagonals, so the result follows from Corollary 4.9.

The even permanent per ${ }^{\mathrm{ev}}$ is defined as the sum of the products of the entries on the even diagonals of a matrix. In other words, it has the same definition as (1) except that the sum is taken over the alternating group rather than the symmetric group. Let $R_{i}^{\text {ev }}$ be defined the same as $R_{i}$, but using per ${ }^{\text {ev }}$ in place of per. Similarly, let $E_{i}^{\mathrm{ev}}$ be the number of even diagonals with exactly $i$ different symbols on them. We considered even permanents as one possible approach to Conjecture 2.13. While that effort was unsuccessful, we did manage to prove this weak analogue of Theorem 4.8:

THEOREM 4.11. If $L$ is a Latin square of even order $n>2$ then

$$
E_{3}^{\mathrm{ev}}+E_{5}^{\mathrm{ev}}+\cdots+E_{n-1}^{\mathrm{ev}} \equiv E_{1}^{\mathrm{ev}}+E_{2}^{\mathrm{ev}}+E_{4}^{\mathrm{ev}}+E_{6}^{\mathrm{ev}}+\cdots+E_{n}^{\mathrm{ev}} \equiv 0 \quad \bmod 2 .
$$

Proof. Similar to the proof of Theorem 4.8, we have that

$$
\sum_{m=1}^{n / 2} E_{2 m-1}^{\mathrm{ev}} \equiv R_{n-1}^{\mathrm{ev}} \quad \bmod 2 .
$$

Hence

$$
\sum_{m=3}^{n / 2} E_{2 m-1}^{\mathrm{ev}} \equiv E_{1}^{\mathrm{ev}}+R_{n-1}^{\mathrm{ev}} \equiv R_{1}^{\mathrm{ev}}+R_{n-1}^{\mathrm{ev}} \quad \bmod 2 .
$$

Let $P_{0}$ and $P_{1}$ be permutation matrices corresponding to arbitrary even and odd permutations, respectively. Then $\operatorname{per}^{\mathrm{ev}} P_{0}=1$ and $\operatorname{per}^{\mathrm{ev}} P_{1}=0$. Also $\operatorname{per}^{\mathrm{ev}}\left(J-P_{0}\right)=$ $a$ and $\operatorname{per}^{\mathrm{ev}}\left(J-P_{1}\right)=b$, where $a+b=\operatorname{per}\left(J-P_{0}\right)=d_{n}$, and $a-b=\operatorname{det}\left(J-P_{0}\right)=1-n$, by (5). Thus $a=\left(d_{n}+1-n\right) / 2$ and $b=\left(d_{n}-1+n\right) / 2$. By (15),

$$
\begin{aligned}
\operatorname{per}^{\mathrm{ev}} P_{0}+\operatorname{per}^{\mathrm{ev}}\left(J-P_{0}\right) & \equiv 1+\left(d_{n}+1-n\right) / 2 \equiv n / 2 \equiv\left(d_{n}-1+n\right) / 2 \\
& \equiv \operatorname{per}^{\mathrm{ev}} P_{1}+\operatorname{per}^{\mathrm{ev}}\left(J-P_{1}\right)
\end{aligned}
$$

mod 2. Thus, in calculating (16) we can pair up complementary terms in $R_{1}^{\text {ev }}$ and $R_{n-1}^{\mathrm{ev}}$ to show that $R_{1}^{\mathrm{ev}}+R_{n-1}^{\mathrm{ev}} \equiv n(n / 2) \equiv 0 \bmod 2$. The result follows, since $\sum_{i} E_{i}^{\mathrm{ev}}=n ! / 2 \equiv 0 \bmod 2$.

Note that $E_{1}^{\mathrm{ev}} \equiv n-\pi_{s} \bmod 2$, where $\pi_{s}$ is the symbol parity described in $\S 1$. It is curious that the $E_{1}^{\mathrm{ev}}$ term in Theorem 4.11 appears on the side of the congruence that it does. The analogous statement for standard permanents follows by considering Theorem 4.8 modulo 2, and noting that the $E_{1}$ term can be written on either side of the congruence, since $E_{1}=n$ is even.

We next show a parity relationship between consecutive pairs in the sequence $E_{1}, \ldots, E_{n}$.

THEOREM 4.12. If $L$ is a Latin square of even order then $E_{2 i-1} \equiv E_{2 i} \bmod 2$ for each integer $i$.

Proof. By (14),

$$
\begin{aligned}
E_{2 i}+E_{2 i-1} & =R_{2 i}+\sum_{r=1}^{2 i-1}\left[\left(\begin{array}{c}
n-r \\
n-2 i
\end{array}\right)-\left(\begin{array}{c}
n-r \\
n-2 i+1
\end{array}\right)\right](-1)^{r} R_{r} \\
& =R_{2 i}+\sum_{r=1}^{2 i-1}\left[\left(\begin{array}{c}
n-r \\
n-2 i
\end{array}\right)-\left(\begin{array}{c}
n-r \\
n-2 i
\end{array}\right)\left(\frac{2 i-r}{n-2 i+1}\right)\right](-1)^{r} R_{r} \\
& =R_{2 i}+\sum_{r=1}^{2 i-1}\left(\begin{array}{c}
n-r \\
n-2 i
\end{array}\right)\left[\frac{n-4 i+r+1}{n-2 i+1}\right](-1)^{r} R_{r} .
\end{aligned}
$$


If $r$ is even, then $R_{r}$ is even. If $r$ is odd, then $n-4 i+r+1$ is even, while $n-2 i+1$ is odd, so $\left(\begin{array}{c}n-r \\ n-2 i\end{array}\right)(n-4 i+r+1) /(n-2 i+1)$ must be even (it is an integer, since our proof shows that it is the difference of two integers). The result follows.

It seems that $E_{2 i}$ and $E_{2 i+1}$ are unrelated except for the case $E_{n-2}$ and $E_{n-1}$ when $n \equiv 0 \bmod 4$, which is covered in the following conjecture.

CONJECTURE 4.13. Let $L$ be a Latin square of order $n$. The following holds for all $i, j$ and $r$. If $n \equiv 0 \bmod 4$, then

$$
E_{n} \equiv E_{n-1} \equiv 2 E_{n-2} \equiv 2 t_{i j} \equiv N_{r} \bmod 4
$$

$$
R_{1}+R_{3}+\cdots+R_{n-1} \equiv 0 \bmod 4 \text { and } R_{2}+R_{4}+\cdots+R_{n} \equiv E_{n} \bmod 4 \text {. }
$$

If $n \equiv 2 \bmod 4$, then $E_{n-1} \equiv 2 t_{i j} \equiv N_{r} \bmod 4$.

Using (14), Theorem 2.7 and Lemma 4.1(d), if $n \equiv 0 \bmod 4$, then $\sum R_{i} \equiv E_{n} \bmod$ 4. Also, $E_{n-1}+2 E_{n-2} \equiv R_{1}+R_{3}+\cdots+R_{n-1} \bmod 4$. Thus, $E_{n-1} \equiv 2 E_{n-2} \bmod 4$ is equivalent to (17). Note, for even $n$, that $E_{n}, E_{n-1}$ and $N_{r}$ are even by Theorem 2.7, Theorem 4.3 and Corollary 3.13, respectively.

Suppose that $n \equiv 0 \bmod 4$ and that Conjecture 4.13 holds. It follows that $N_{r}+E_{n} \equiv$ 0 mod 4, which combines with Lemma 3.10 to imply that the number of transversals in any $(n-1) \times n$ Latin rectangle is divisible by 4 , whenever $n$ itself is divisible by 4 .

Our results to this point have all been congruences mod 2 or 4 . We finish by showing for any given order $n$ that $R_{2}$ and $E_{2}$ have only two possible values mod 6 . The main interest in this result is that it involves a different modulus to our other results.

LEMmA 4.14. Let $L$ be a Latin square of any order $n$. Then $R_{2} \equiv E_{2} \equiv 0 \bmod 2$ and

$$
R_{2} \not \equiv(-1)^{n}(n+1) \bmod 3 \text { and } E_{2} \not \equiv(-1)^{n}(n+1)-n(n-1) \bmod 3 .
$$

Proof. By (14) we have that $E_{2}=R_{2}-(n-1) R_{1}=R_{2}-n(n-1)$, so it suffices to prove the claims about $R_{2}$. By Lemma $4.1(\mathrm{~d})$, we know that $R_{2}$ is even, and it follows immediately that $E_{2}$ is even as well.

For each symbol $s$ of $L$, define a permutation $\theta_{s}:[n] \rightarrow[n]$ by $\theta_{s}(i)=j$ if $L_{i j}=s$. Then $R_{2}$ is the sum over symbols $s, s^{\prime} \in[n]$ of $2^{c\left(s, s^{\prime}\right)}$, where $c\left(s, s^{\prime}\right)$ is the number of cycles in $\left(\theta_{s}\right)^{-1} \theta_{s^{\prime}}$. The number of cycles in any permutation $\sigma \in \mathcal{S}_{n}$ is $n-\varepsilon(\sigma) \bmod 2$. Hence

$$
\begin{aligned}
R_{2} & =\sum_{s, s^{\prime}} 2^{c\left(s, s^{\prime}\right)} \equiv \sum_{s, s^{\prime}}(-1)^{n-\varepsilon\left(\left(\theta_{s}\right)^{-1} \theta_{s^{\prime}}\right)} \equiv(-1)^{n} \sum_{s, s^{\prime}}(-1)^{\varepsilon\left(\theta_{s}\right)+\varepsilon\left(\theta_{s^{\prime}}\right)} \\
& \equiv(-1)^{n}\left(\left(\begin{array}{l}
e \\
2
\end{array}\right)+\left(\begin{array}{c}
n-e \\
2
\end{array}\right)-e(n-e)\right) \bmod 3
\end{aligned}
$$

where $e=\left|\left\{s \in[n]: \varepsilon\left(\theta_{s}\right)=0\right\}\right|$. The required result now follows by a simple case analysis concerning the value of $e \bmod 3$.

\section{CONCLUDing REMARKS}

We have shown a number of congruences satisfied by various quantities motivated by the study of transversals in Latin squares. There are many others which are direct consequences of the results we have given. For example, it is easy to use (14), Lemma $4.1(\mathrm{~d})$ and Corollary 4.4 to show that $E_{8} \equiv 0 \bmod 4$ when $n \equiv 3 \bmod 4$, given 
that

$$
\begin{aligned}
& \left(\begin{array}{c}
n-7 \\
1
\end{array}\right) \equiv\left(\begin{array}{c}
n-5 \\
3
\end{array}\right) \equiv\left(\begin{array}{c}
n-3 \\
5
\end{array}\right) \equiv\left(\begin{array}{c}
n-2 \\
6
\end{array}\right) \equiv\left(\begin{array}{c}
n-1 \\
7
\end{array}\right) \equiv 0 \bmod 4 \text { and } \\
& \left(\begin{array}{c}
n-6 \\
2
\end{array}\right) \equiv 0 \bmod 2 .
\end{aligned}
$$

As mentioned in the introduction, a notion of parity has been useful in a number of different studies of Latin squares. In this paper we have introduced parity for transversals of Latin squares, and used it in the analysis of the number of transversals. In our investigation we uncovered a number of interesting patterns, some of which we have proved, and others we conjecture. Several of the conjectures classify Latin squares of a given even order into two types which seem to have different properties. These classifications do not seem to be related to each other, or to pre-existing notions of parity. In Conjecture 2.13 the value of $w \bmod 2$, say, partitions Latin squares based on the parity of their transversals, while Conjecture 4.13 partitions Latin squares into two classes based on $E_{n-1}$ modulo 4 . However, these partitions seem to be independent of each other and of the previously studied parities $\pi_{r}$ and $\pi_{c}$. By randomly generating Latin squares, we found a Latin square with each of the 16 possibilities for $\left(w, E_{n-1} / 2, \pi_{r}, \pi_{c}\right) \bmod 2$ for orders 8,10 and 12 .

Finally, we remark that we have only considered the classical 2-dimensional case in this paper. However, transversals are of interest in the context of Latin hypercubes and permanents can also be generalised to higher dimensions. All of the questions that we have investigated could also be asked in these higher dimensional contexts. A first step in this direction has been taken by Taranenko [24], who noted that Theorem 2.7 generalises to Latin hypercubes.

Acknowledgements. The authors are grateful to Saieed Akbari for interesting discussions on the topic of this paper.

\section{REFERENCES}

[1] Ron Aharoni and Martin Loebl, The odd case of Rota's bases conjecture, Adv. Math. 282 (2015), 427-442.

[2] Saieed Akbari and Alireza Alipour, Transversals and multicolored matchings, J. Comb. Des. 12 (2004), no. 5, 325-332.

[3] Robert E. L. Aldred, Rosemary A. Bailey, Brendan D. McKay, and Ian M. Wanless, Circular designs balanced for neighbours at distances one and two, Biometrika 101 (2014), no. 4, 943-956.

[4] Noga Alon and Michael Tarsi, Colorings and orientations of graphs, Combinatorica 12 (1992), no. 2, 125-134.

[5] Levent Alpoge, Square-root cancellation for the signs of Latin squares, Combinatorica 37 (2017), no. 2, 137-142.

[6] Krishnaswami Balasubramanian, On transversals in Latin squares, Linear Algebra Appl. 131 (1990), 125-129.

[7] János Barát and Zoltán L. Nagy, Transversals in generalized Latin squares, Ars Math. Contemp. 16 (2019), no. 1, 39-47.

[8] Darcy Best, Kevin Hendrey, Ian M. Wanless, Tim E. Wilson, and David R. Wood, Transversals in Latin arrays with many distinct symbols, J. Comb. Des. 26 (2018), no. 2, 84-96.

[9] Nicholas J. Cavenagh and Ian M. Wanless, There are asymptotically the same number of Latin squares of each parity, Bull. Aust. Math. Soc. 94 (2016), no. 2, 187-194.

[10] Diane M. Donovan, Michael J. Grannell, Terry S. Griggs, and James G. Lefevre, On parity vectors of Latin squares, Graphs Comb. 26 (2010), no. 5, 673-684.

[11] Nevena Francetić, Sarada Herke, and Ian M. Wanless, Parity of sets of mutually orthogonal Latin squares, J. Comb. Theory, Ser. A 155 (2018), 67-99.

[12] David G. Glynn, The conjectures of Alon-Tarsi and Rota in dimension prime minus one, SIAM J. Discrete Math. 24 (2010), no. 2, 394-399.

[13] David G. Glynn and David Byatt, Graphs for orthogonal arrays and projective planes of even order, SIAM J. Discrete Math. 26 (2012), no. 3, 1076-1087. 
[14] Jeannette C. M. Janssen, On even and odd Latin squares, J. Comb. Theory, Ser. A 69 (1995), no. 1, 173-181.

[15] Petteri Kaski, André de Souza Medeiros, Patric R. J. Östergård, and Ian M. Wanless, Switching in one-factorisations of complete graphs, Electron. J. Comb. 21 (2014), no. 2, Paper \#2.49 (23 pages).

[16] Peter Keevash and Liana Yepremyan, On the number of symbols that forces a transversal, to appear in Comb. Probab. Comput., 7 pages.

[17] Daniel Kotlar, Parity types, cycle structures and autotopisms of Latin squares, Electron. J. Comb. 19 (2012), no. 3, Paper \#10 (17 pages).

[18] Brendan D McKay, Jeanette C. McLeod, and Ian M. Wanless, The number of transversals in a Latin square, Des. Codes Cryptography 40 (2006), no. 3, 269-284.

[19] Richard Montgomery, Alexey Pokrovskiy, and Benjamin Sudakov, Decompositions into spanning rainbow structures, Proc. Lond. Math. Soc. (3) 119 (2019), no. 4, 899-959.

[20] Morris Newman, Combinatorial matrices with small determinants, Can. J. Math. 30 (1978), no. 4, 756-762.

[21] Herbert J. Ryser, Combinatorial mathematics, Carus Math. Monogr., vol. 14, Wiley, 1963.

[22] _ Neuere Probleme der Kombinatorik, Vortrage über Kombinatorik Oberwolfach, July 1967, pp. 69-91.

[23] Douglas S. Stones and Ian M. Wanless, How not to prove the Alon-Tarsi conjecture, Nagoya Math. J. 205 (2012), 1-24.

[24] Anna A. Taranenko, Permanents of multidimensional matrices: properties and applications, J. Appl. Ind. Math. 10 (2016), no. 4, 567-604.

[25] Ian M. Wanless, Cycle switches in Latin squares, Graphs Comb. 20 (2004), no. 4, 545-570.

[26] _. Transversals in Latin squares: a survey, in Surveys in combinatorics 2011, London Math. Soc. Lecture Note Ser., vol. 392, Cambridge Univ. Press, Cambridge, 2011, pp. $403-437$.

DARCY Best, School of Mathematics, Monash University, Australia

IAn M. WAnless, School of Mathematics, Monash University, Australia

E-mail : ian.wanless@monash.edu 